\title{
Моделирование туннельного переноса электронов в системе полупроводник-кристаллический диэлектрик-Si(111)
}

\author{
() М.И. Векслер
}

Физико-технический институт им. А.Ф. Иофрфе Российской академии наук, 194021 Санкт-Петербург, Россия

E-mail: vexler@mail.ioffe.ru

(Получена 11 декабря 2017 г. Принята к печати 12 декабря 2017 г.)

Теоретически исследован туннельный транспорт носителей через тонкий слой диэлектрика (для примера рассмотрен $\mathrm{CaF}_{2}$ ) между кремниевой подложкой ориентации (111) и полупроводниковым затвором. Наряду с сохранением большого по величине поперечного волнового вектора туннелирующих частиц учтено ограничение на наличие состояний в затворе. Из-за этого ограничения туннельные токи при малых напряжениях на диэлектрике оказываются меньше, чем в аналогичной структуре с металлическим затворным электродом. Эта же особенность обусловливает изменение вида энергетического распределения туннелирующих электронов, причем как при транспорте между зонами проводимости подложки и затвора, так и при переносе зона проводимости $\mathrm{Si}(111)$ - валентная зона затвора.

DOI: $10.21883 /$ FTP.2018.08.46216.8753

\section{1. Введение}

К настоящему времени теория структур металл/полупроводник-диэлектрик-полупроводник (МДП, ПДП) с туннельно-тонким диэлектриком разработана весьма тщательно [1,2]. Однако в подавляющем большинстве случаев рассматриваемый слой изолятора является или считается аморфным ( $\mathrm{SiO}_{2}, \mathrm{HfO}_{2}$ и др.) [2,3] и предполагается, часто даже без указания данного факта, что структура выполнена на кремниевой подложке ориентации (100).

Согласно теории, при туннелировании электрона его полная энергия $E$ и поперечная компонента волнового вектора $k_{\perp}$ остаются неизменными. Условие сохранения $k_{\perp}$ должно приводить к радикальному различию вероятностей туннельного прохождения между случаями $\mathrm{Si}(100)$ и $\mathrm{Si}(111)$ из-за большого сдвига $k_{0}$ минимума зоны проводимости кремния от центра зоны Бриллюэна. Если при ориентации (100) наличие этого сдвига непринципиально, так как в двух из шести $(v=6)$ эквивалентных долин значения $k_{\perp}^{2}$ у электронов вблизи энергетического минимума невелики, то для (111) они должны составлять $\sim 2 / 3 k_{0}^{2}$ во всех долинах. Однако при экспериментальных исследованиях структур с оксидами не отмечалось заметных изменений туннельных токов при смене ориентации [1] и поэтому для расчетов всегда применялась „модель $k_{0}=0$ “.

Относительно недавно при изучении МДП-структур с эпитаксиальным кристаллическим фторидом кальция $\left(\mathrm{CaF}_{2}\right)$, выращенным на подложке $\mathrm{Si}(111)$, были зарегистрированы намного меньшие токи, чем предсказывалось „моделью $k_{0}=0^{“}$ соответственно была подтверждена реальность сохранения $k_{\perp}[4,5]$. В исследованных в цитированных работах образцах в качестве верхнего электрода (условно называемого „затвором“) использовался слой золота.

Обычно предполагается, что со стороны металла не появляется никаких ограничений на диапазон парамет- ров $E, k_{\perp}$ частиц, которые могут участвовать в туннелировании: там настолько много разных состояний, что все ограничения на возможность транспорта связаны исключительно с подложкой. Но на практике в роли затвора вместо металла нередко выступает полупроводниковый материал - так, применительно к системе $\mathrm{CaF}_{2} / \mathrm{Si}(111)$ поверх фторида, в принципе могут быть нанесены слои GaAs или графена. Поэтому в настоящей работе будет теоретически рассмотрена не МДП-, а ПДП-структура. В отличие от подложки, „затворный“ полупроводник предполагается прямозонным, с изотропными массами $m_{\mathrm{cG}}, m_{\mathrm{vG}}$ в обеих зонах. Задачей данной работы будет определение особенности токопереноса в подобной структуре в рамках простейших предположений.

На качественном уровне очевидно, что указанная замена материала затвора должна приводить к снижению туннельного тока, поскольку плотность доступных состояний в случае полупроводника меньше. Однако без выполнения расчетов невозможно ни оценить количественную сторону этого эффекта, ни выявить условия сравнительно более сильного или более слабого спада тока, ни сделать какие-либо выводы относительно влияния смены материала на энергетическое распределение туннелирующих носителей. Вместе с тем названные детали весьма важны для понимания поведения структур с кристаллическими диэлектриками на подложках $\mathrm{Si}(111)$; ясно также, что предварительный теоретический анализ полезен для последующих попыток изготовления реальных образцов.

\section{2. Типы транспорта в изучаемой системе}

На рис. 1 представлены схемы возможного энергетического соположения краев зон проводимости и валентных зон в изучаемой системе. Для простоты изгибы зон в $\mathrm{Si}(111)$ и в затворе игнорируются. Очевидно, что 


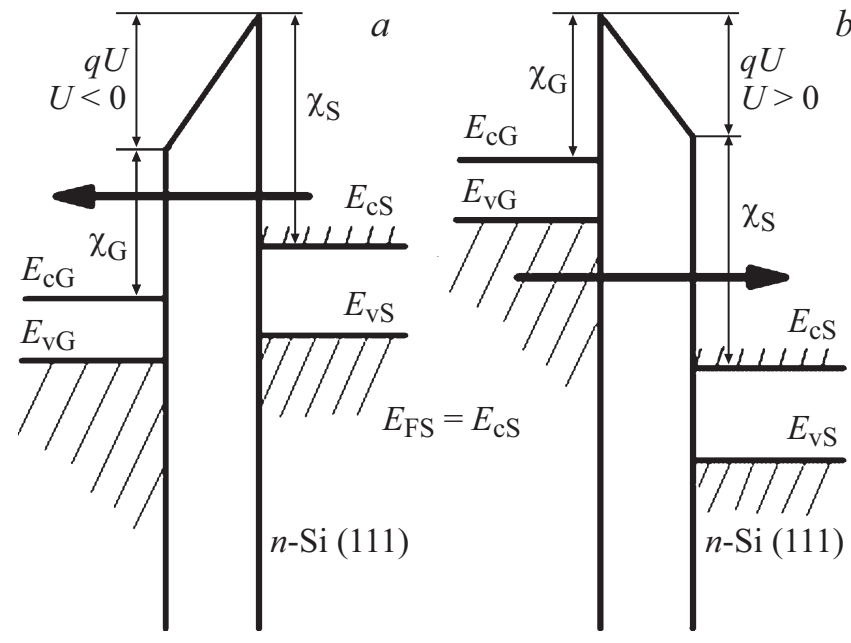

Рис. 1. Зонные диаграммы изучаемых структур с принятыми обозначениями: $a-U<0 ; b-U>0$. Отмечены возможные варианты туннельного переноса электронов. Для простоты изгиб зон в $\mathrm{Si}(111)$ и в полупроводниковом затворе игнорируется; уровень Ферми в подложке $E_{\mathrm{FS}}$ считается совпадающим с краем зоны.

может иметь место перенос типа „с-с“ между зонами проводимости обеих частей (левая диаграмма) и перенос „V-c“ между валентной зоной затвора и зоной проводимости подложки. Эти типы транспорта помечены стрелкой (вообще говоря, туннелирование можно трактовать и как перенос электронов, и как перенос дырок в противоположную сторону; направления стрелок на рисунках отвечают электронам). Перенос с участием валентной зоны Si не рассматривается: он, безусловно, возможен, но максимум этой зоны находится в Г-точке при любой ориентации подложки, и поэтому такой перенос не представляет новизны.

\section{3. Вероятность туннелирования в случае $\mathrm{Si}(111)$}

Как известно, вероятность туннелирования частицы с полной энергией $E$ и поперечным волновым вектором $k_{\perp}$, в приближении Вентцеля-Крамерса-Бриллюэна (ВКБ), записывается [1]:

$$
T=\exp \left[-\frac{2 \sqrt{2 m_{i}}}{\hbar} \int \sqrt{E_{\mathrm{c} i}(z)-E+\frac{\hbar^{2} k_{\perp}^{2}}{2 m_{i}}} d z\right],
$$

где через $E_{\mathrm{c} i}(z)$ обозначен профиль зоны проводимости диэлектрического барьера, а $m_{i}-$ масса частицы в барьере. При этом предполагается, что диэлектрический барьер имеет настолько широкую запрещенную зону, что взаимодействие частицы с валентной зоной невозможно (для $\mathrm{CaF}_{2}$ это именно так: ширина зоны равна 12.1 эВ [6]). Данное выражение применимо во всех случаях, а особенности зонной структуры эмиттера отразятся в записи $k_{\perp}$.
Строго говоря, перед ВКБ-экспонентой в (1) нужен корректирующий фактор $T_{\mathrm{R}}$ (почти всегда опускаемый), содержащий комбинацию $z$-компонент групповых скоростей частицы у гетерограниц вне и внутри барьера [7]. Этим фактором обеспечивается подход $T$ к нулю вблизи краев энергетических диапазонов, доступных для туннелирования, но на точность последующего вычисления тока отсутствие $T_{\mathrm{R}}$ влияет мало (важнее выбор $m_{i}$ и разрывов зон), так как роль $T_{\mathrm{R}}$ нивелируется интегрированием по состояниям.

Кремний является непрямозонным полупроводником, шесть эквивалентных долин зоны проводимости которого (см. вставку на рис. 2) расположены в направлениях (100) на расстоянии в $k$-пространстве $k_{0}=0.85 k_{b}$, где $k_{b}=2 \pi / a_{\mathrm{L}}\left(a_{\mathrm{L}}=0.543\right.$ нм - постоянная решетки $)$.

Волновой вектор электрона в одной из шести эквивалентных долин составляет

$$
\mathbf{k}=\left(k_{0}+k_{l}\right) \mathbf{e}_{l}+k_{r} \cos \varphi \mathbf{e}_{t 1}+k_{r} \sin \varphi \mathbf{e}_{t 2},
$$

где единичный орт $e_{l}$ выбран вдоль некоторого направления (100), а два других орта — в перпендикулярной плоскости (тоже вдоль направлений (100)), $k_{l}$ и $k_{r}$ суть составляющие волнового вектора вдоль главной оси эллипсоида и в указанной перпендикулярной ей плоскости ( $\varphi$ - угол в ней).

Для квадрата поперечного волнового вектора можно получить (см. Приложсение):

$k_{\perp}^{2}=\frac{2\left(k_{0}+k_{l}\right)^{2}}{3}\left[1+a^{2}-a(\cos \varphi+\sin \varphi)-a^{2} \cos \varphi \sin \varphi\right]$,

где

$$
a=\frac{k_{r}}{k_{0}+k_{l}} .
$$

Для любой изоэнергетической поверхности в $k$-пространстве (т.е. для любой заданной энергии $E$ ) при известном $k_{l}$ автоматически определяется $k_{r}$, а именно

$$
k_{r}=\sqrt{\frac{2 m_{t}\left(E-E_{\mathrm{cS}}\right)}{\hbar^{2}}-\frac{m_{t}}{m_{l}} k_{l}^{2}} .
$$

В этой формуле $m_{l}=0.916 m_{0}, m_{t}=0.191 m_{0}-$ продольная и поперечная эффективные массы электрона в $\mathrm{Si}$, причем $k_{l}$ может лежать в пределах $-k_{l m} \ldots+k_{l m}$ $\left(k_{l m}=\left[2 m_{l}\left(E-E_{\mathrm{cS}}\right]^{1 / 2} / \hbar\right)\right.$, где $E_{\mathrm{cS}}-$ энергия края зоны проводимости $\mathrm{Si}(111)$ у его границы с диэлектриком (в принципе, ее можно принять за нуль).

Соответственно вероятность туннелирования $T$ оказывается выраженной через $E, k_{l}$ и $\varphi$.

\section{4. Возможность туннельного процесса}

Анализируя выражение для $k_{\perp}^{2}$, можно проверить, что оно принимает - для данных $k_{l}, k_{r}-$ наименьшие значения при $\sin \varphi=\cos \varphi= \pm 2^{-1 / 2}$. Дифференцируя 


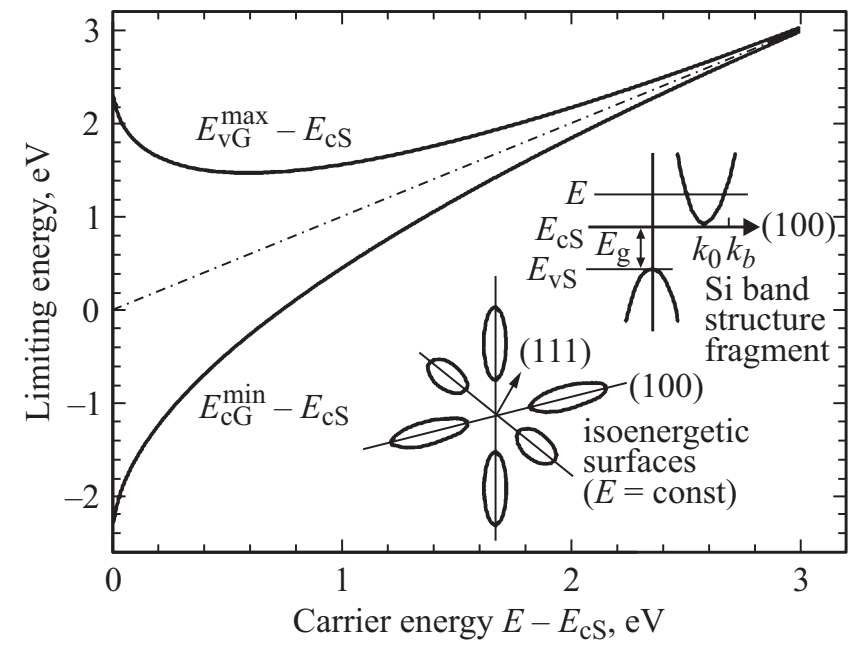

Рис. 2. Ограничение на положения краев зон $E_{\mathrm{vG}}$ и $E_{\mathrm{cG}}$, при которых возможен транспорт носителей с энергией $E$ между $\mathrm{Si}(111)$ и затвором по одному из механизмов, показанных на рис. 1. Для ориентации (100) кривые слились бы в наклонную пунктирную прямую. На вставке - фрагменты зонной структуры кремния.

$k_{\perp}^{2}$ при этом условии по $k_{l}$, а затем выражая $k_{l}$ через $E$, находим минимум:

$$
\left(k_{\perp}^{2}\right)_{\min }=\frac{2}{3}\left[k_{0}-\frac{\sqrt{\left(m_{t}+2 m_{l}\right)\left(E-E_{\mathrm{cS}}\right)}}{\hbar}\right]^{2} .
$$

Если $E_{\mathrm{cG}}$ - край зоны проводимости затвора, то туннельный процесс, „с-с“ между зонами проводимости возможен только при выполнении соотношения $\left(m_{\mathrm{cG}}-\right.$ масса электрона в затворе, принимаемая изотропной):

$$
E-\frac{\hbar^{2}\left(k_{\perp}^{2}\right)_{\min }}{2 m_{\mathrm{cG}}} \geq E_{\mathrm{cG}}
$$

Аналогично, если $E_{\mathrm{vG}}$ - край валентной зоны затвора, туннельный процесс „,-c“ между зоной проводимости $\mathrm{Si}(111)$ и валентной зоной затвора осуществляется при $\left(m_{\mathrm{vG}}\right.$ - масса электрона в затворе $)$

$$
E+\frac{\hbar^{2}\left(k_{\perp}^{2}\right)_{\min }}{2 m_{\mathrm{vG}}} \geq E_{\mathrm{vG}} .
$$

Два последних ограничения через $E_{\mathrm{cG}}$ и $E_{\mathrm{vG}}$ были неактуальными в случае туннелирования электронов в металл, где нет своей запрещенной зоны (так как $E_{\mathrm{cG}}$ и тем более $E_{\mathrm{VG}}$ лежат очень низко по диаграмме).

На рис. 2 для системы полупроводник- $\mathrm{CaF}_{2}-\mathrm{Si}(111)$ построены расчетные зависимости минимального $E_{\mathrm{vG}}$ или максимального значения $E_{\mathrm{cG}}$, необходимые для создания возможности туннелирования электрона с энергией $E$ между затвором и подложкой по типу „с-с“ или „V-c“ соответственно. Значения эффективных масс положены равными единице $\left(m_{\mathrm{cG}}=m_{\mathrm{vG}}=1\right)$. Из всех энергий вычитается „опорное“ значение $E_{\mathrm{cS}}$. В случае металлического затвора никаких ограничений не возникало бы вообще, а если бы кремний был прямозонным, то $E, E_{\mathrm{cG}}^{\min }$ и $E_{\mathrm{vG}}^{\max }$ совпали бы (наклонный пунктир на рисунке), так как при любом $E$ в $\mathrm{Si}$ существовало бы состояние с нулевым $k_{\perp}$.

\section{5. Туннельный ток в изучаемой системе}

Вычисление туннельного тока предполагает интегрирование вероятности $T$ по энергии $E$, а также по поперечным компонентам волнового вектора $k_{x}, k_{y}$. Так как у нас аргументами $T$ выступают $k_{l}, \varphi$, потребуется якобиан преобразования (см. Приложсение):

$$
|J|=\frac{k_{r}}{\sqrt{3}}\left[\sin \varphi+\cos \varphi+\frac{m_{t} k_{l}}{m_{l} k_{r}}\right] .
$$

Это позволяет записать выражение для туннельного тока как

$$
j=\frac{q v}{4 \pi^{3} \hbar}|\Delta f d E| T\left(E, k_{\perp}^{2}\right)|J| d k_{l} d \varphi
$$

(в традиционной записи на месте $|J| d k_{l} d \varphi$ стоит $d k_{x} d k_{y}$ - в случае параболического закона дисперсии и прямозонного полупроводника мог осуществляться переход $d k_{x} d k_{y}=2 \pi k_{r} d k_{r}=2 \pi m_{\perp} \hbar^{-2} d E_{\perp}$ к часто используемой формуле $j=4 \pi q h^{-3} v m_{\perp} \int \Delta f d E \int T\left(E, E_{\perp}\right) d E_{\perp}$, которая в данном случае $(\mathrm{Si}(111))$ неприменима. Через $\Delta f$ обозначена разность функций Ферми на двух сторонах барьера.

Выражение для тока $j$, выписанное выше, применимо к переносу и в зону проводимости затвора, и в его валентную зону (изменятся только диапазоны по $E$ ). При вычислениях, если нужно проверить возможность туннелирования конкретного электрона, в выражения предыдущего раздела, лимитирующие возможность транспорта, следует подставить $k_{\perp}^{2}$ именно для этого электрона, вместо $\left(k_{\perp}^{2}\right)_{\min }$.

Представленная выше модель ранее успешно применялась для расчета туннельных токов в структурах $\mathrm{Au} / \mathrm{CaF}_{2} / \mathrm{Si}(111)$ [5], в которых, как уже констатировалось, нет ограничений на возможность туннелирования, обусловленных металлом.

\section{6. Примеры расчетов и обсуждение}

Для общей иллюстрации особенностей переноса электронов в системе полупроводник-кристаллический диэлектрик- $\mathrm{Si}(111)$ мы примем набор параметров, не привязываясь к конкретному сочетанию материалов, но, конечно, в реалистичном диапазоне. А именно положим эффективные массы $m_{i}, m_{\mathrm{cG}}, m_{\mathrm{vG}}$ равными $m_{0}$, разрыв $\chi_{\mathrm{S}}$ зоны проводимости на границе $\mathrm{Si}(111)$ и диэлектрика приравняем 2.38 эВ (соответствует интерфейсу $\left.\mathrm{Si} / \mathrm{CaF}_{2}[6]\right)$, разность $E_{\mathrm{cG}}-E_{\mathrm{vG}}=1.0$ эB, а толщина слоя 

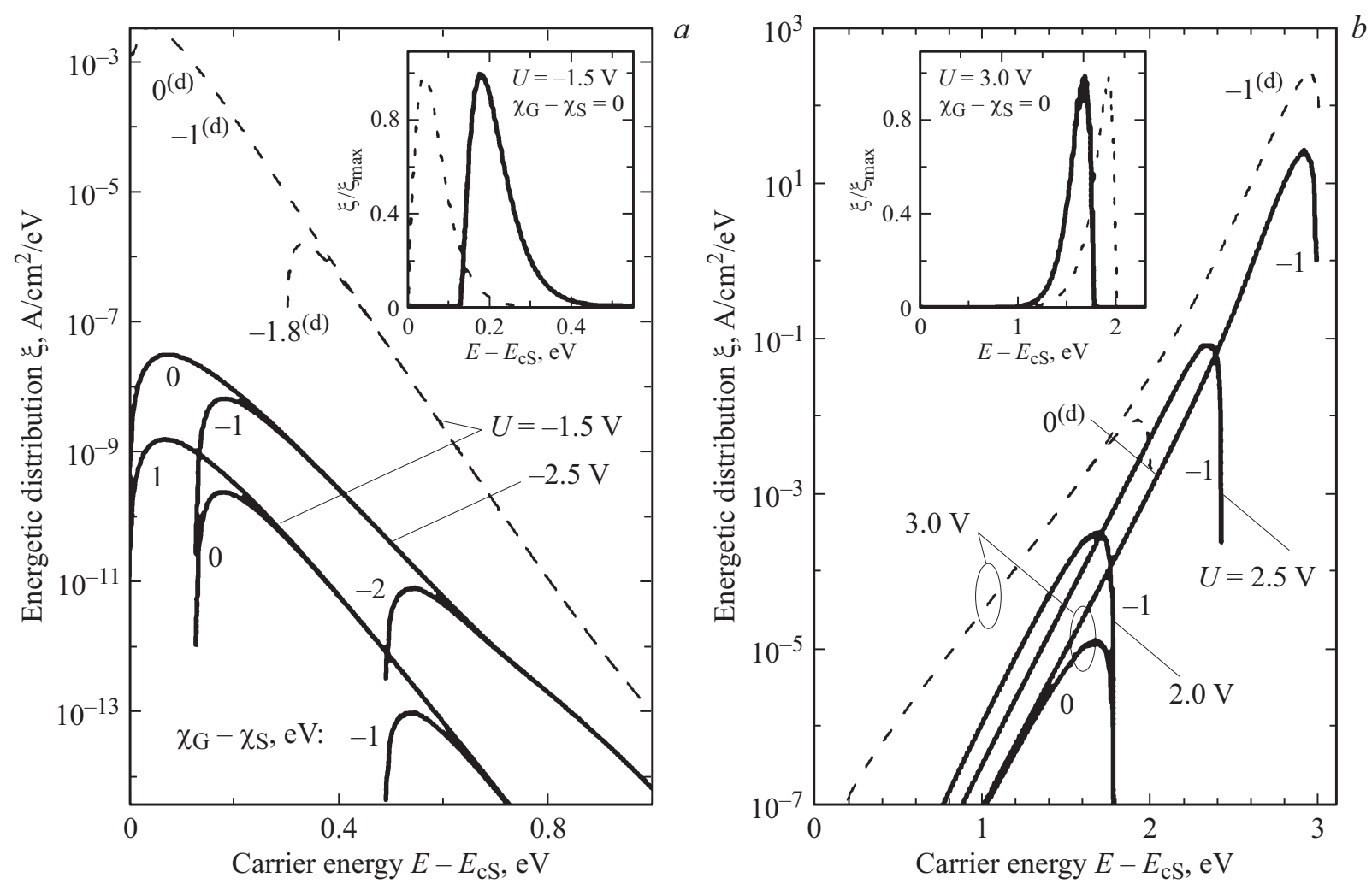

Рис. 3. Рассчитанные энергетические распределения электронов для условий транспорта, показанных на рис. $1: a-U<0$; $b-U>0$. Цифрами помечена разность (в эВ) высот барьера. Для сравнения пунктиром построены распределения в модели $k_{0}=0$ (пометка „đ“). На вставках — примеры тех же кривых с делением на максимум.
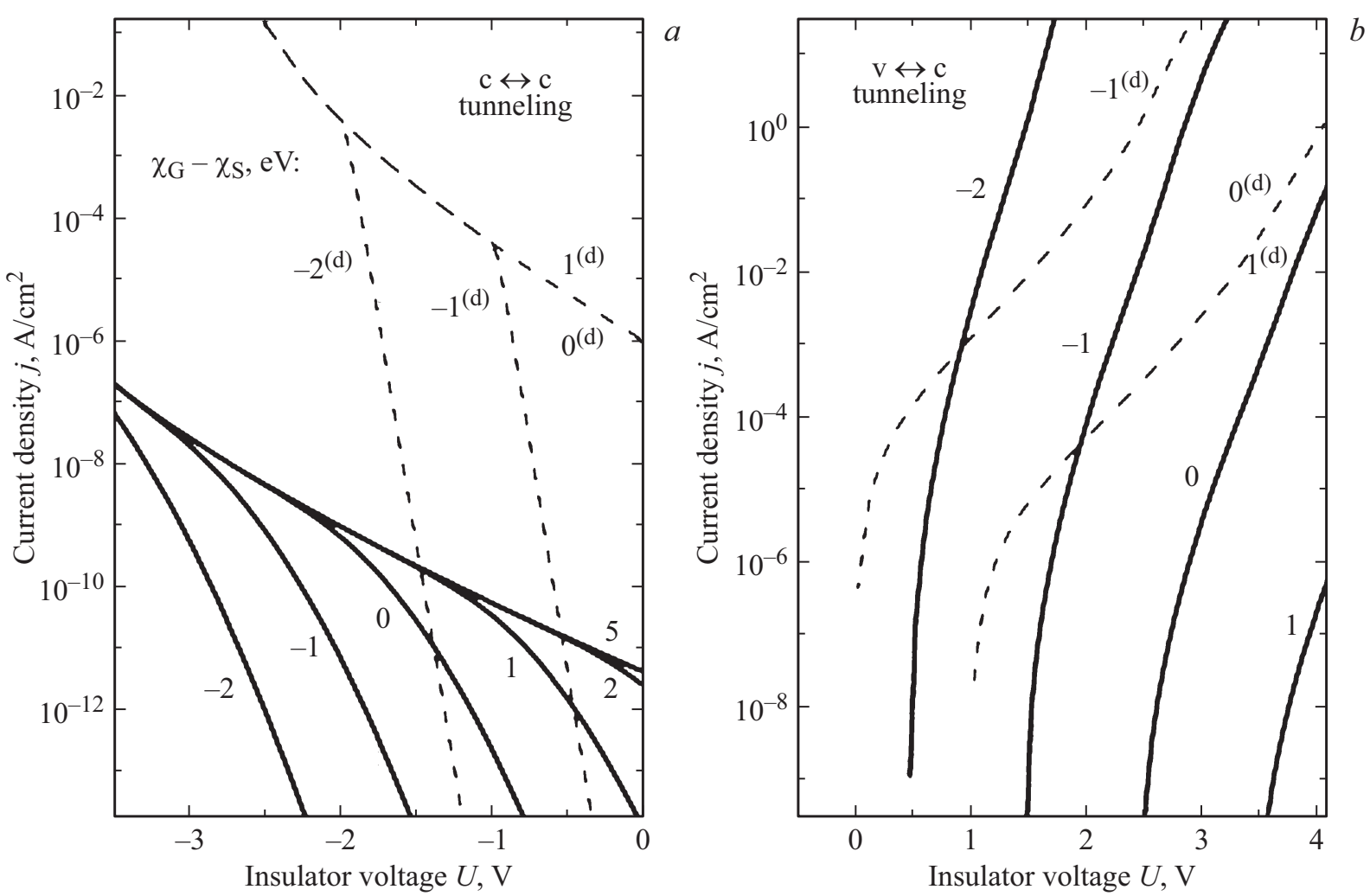

Рис. 4. Рассчитанные туннельные токи для условий рис. $1: a-U<0 ; b-U>0$. Сплошные кривые получены с учетом зонной структуры в случае $\mathrm{Si}(111)$, а пунктирные - в модели $k_{0}=0$. На рис. $a$ большое положительное значение разности $\chi_{\mathrm{G}}-\chi_{\mathrm{S}}$ имитирует ситуацию металлического затвора (по сравнению с ней токи при низких $|U|$ резко уменьшаются в случае полупроводникового затвора). 
диэлектрика $d=2$ нм. Было проверено, что варьирование параметров не влечет качественных изменений.

Далее будут выполнены расчеты распределений туннелирующих электронов по энергии, а также туннельных токов при нескольких значениях разности разрывов зоны проводимости (111) кремний-диэлектрик $\chi_{\mathrm{S}}$ и затвор-диэлектрик $\chi_{\mathrm{G}}$. При этом, естественно,

$$
\left(E_{\mathrm{cG}}-E_{\mathrm{cS}}\right)=\left(\chi_{\mathrm{S}}-\chi_{\mathrm{G}}\right)+U
$$

где $U$ - напряжение на диэлектрике. Положение уровня Ферми $E_{\mathrm{FS}}$ в $\mathrm{Si}(111)$ принимается совпадающим с краем зоны проводимости $E_{\mathrm{cS}}$, что является вполне реальной ситуацией. Валентную зону затвора считаем полностью заполненной электронами (т.е. функция Ферми $f_{\mathrm{G}}$ там тождественно равна 1 ), а его зона проводимости считается пустой $\left(f_{\mathrm{G}}=0\right)$.

На рис. 3 показаны распределения тока по энергии, т.е. величина $\xi(E)$, подлежащая интегрированию $\left(j=\int \xi d E\right)$ по $E$ в формуле (10), для нескольких ситуаций. На вставках рис. 3 показана та же величина взвешеная на ее максимальное значение. Пунктиром построены аналогичные кривые в ,прямозонной“ $\left(k_{0}=0\right.$, помечено „d“ = direct) модели; при этом значения $\xi(E)$ намного больше, - но это легко понятное и не единственное различие. Более интересно то, что максимум распределения, получаемого с учетом $k_{0} \neq 0$, явно смещен по сравнению с приближением $k_{0}=0$, как это видно на обоих рисунках. Помимо того, кривые могут не начинаться от нуля, а стартовать заметно правее, что также связано с необходимостью обеспечить сохранение поперечного волнового вектора $k_{\perp}$ и наличие доступных состояний слева и справа от барьера.

На рис. 4 представлены рассчитанные зависимости тока от напряжения. Имеются явные количественные различия с прямозонной моделью, но интереснее проанализировать иной аспект, а именно отличие от случая металлического затвора. Этому случаю соответствует большая разность $\chi_{\mathrm{G}}-\chi_{\mathrm{S}}$, т. е. ситуация, когда край $E_{\mathrm{cG}}$ расположен низко. На рис. 4, $a$ видно, что по сравнению с такой ситуацией, при значениях упомянутой разности около или меньше нуля токи при низких напряжениях на диэлектрике $|U|$ ощутимо - на несколько порядков величины - снижаются. Что касается рис. $4, b$, то эти данные не могут быть сопоставлены со случаем металла, так как межзонный перенос „, $-\mathrm{c}^{\text {c }}$ осуществим только для полупроводникового затвора.

\section{7. Комментарий к результату}

Последняя установленная особенность - снижение тока при низких напряжениях за счет использования полупроводникового (а не металлического) затвора может иметь серьезное прикладное значение. Как известно, одной из актуальных задач в сфере МДПструктур является минимизация туннельной утечки [3].
Это принципиально для полевых транзисторов, в которых сечение „затвор-подложка“ представляет собой не что иное, как МДП(ПДП)-структуру. Наиболее традиционный способ снижения утечки предполагает применение изоляторов с высокой диэлектрической постоянной. Полученный результат (рис. 4) показывает, что при определенных обстоятельствах использование именно полупроводникового материала в качестве затвора тоже может способствовать снижению туннельной утечки.

В пределе больших разностей $\chi_{\mathrm{G}}-\chi_{\mathrm{S}}>0$ значения токов для механизма ,с-c $\mathrm{c}^{\text {“ }}$ на рис. 4, $a$ примерно согласуются с ранее опубликованными результатами для системы с металлом на $\mathrm{CaF}_{2} / \mathrm{Si}(111)$ [4,5]. Однако подчеркнем, что цель настоящей работы состояла не в воспроизведении каких-либо данных, а в выявлении следствий замены металлического затвора на полупроводниковый.

Необходимо отметить, что эти следствия не сводятся просто к общему снижению тока $j$ из-за меньшей плотности состояний в полупроводниковом затворе. На самом деле снижение $j$ происходит крайне неодинаково в различных условиях (рис. 4,a): если при напряжениях $|U|$ около нуля оно весьма значительно, то при повышении $|U|$ роль смены материала затвора постепенно сходит на нет при любых $\chi_{\mathrm{G}}$ и $\chi_{\mathrm{S}}$. Кроме того, при переходе разности $\chi_{\mathrm{G}}-\chi_{\mathrm{S}}$ к отрицательным значениям, т. е. при отдалении от случая металла, максимум инжекции электронов типа „с-с“" в ПДП-системе сдвигается к более высоким энергиям (рис. 3). Упомянутые детали актуальны для анализа функционирования приборов затвор-кристаллический диэлектрик-Si(111).

\section{8. Заключение}

В работе было выполнено моделирование туннельного транспорта электронов в ПДП-структуре на положке $\mathrm{Si}(111)$. Одновременно учтены две особенности: необходимость сохранения поперечной компоненты волнового вектора $k_{\perp}$ при туннелировании и наличие запрещенной зоны в полупроводниковом затворе. Модель актуальна в первую очередь для случая кристаллического диэлектрика, когда указанное сохранение должно строго выполняться. Дополнительные и, как выясняется, весьма серьезные ограничения связаны с наличием в затворе области недоступных энергий (этим рассмотренная система отличается от системы с металлическим затвором). Показано, что последнее обстоятельство резко снижает предсказываемые токи при малых напряжениях на диэлектрике.

\section{Приложение}

Для ориентации $\mathrm{Si}(111)$ орты $e_{l}, e_{t 1}, e_{t 2}$, фигурирующие в выражении (2) основного текста, можно связать с ортами, задающими ориентацию подложки, следующим 
образом:

$$
\begin{gathered}
\mathbf{e}_{z}=\frac{1}{\sqrt{3}} \mathbf{e}_{l}+\frac{1}{\sqrt{3}} \mathbf{e}_{t 1}+\frac{1}{\sqrt{3}} \mathbf{e}_{t 2}, \\
\mathbf{e}_{x}=\frac{1}{\sqrt{2}} \mathbf{e}_{l}-\frac{1}{\sqrt{2}} \mathbf{e}_{t 1}, \\
\mathbf{e}_{y}=\frac{1}{\sqrt{6}} \mathbf{e}_{l}+\frac{1}{\sqrt{6}} \mathbf{e}_{t 1}-\frac{2}{\sqrt{6}} \mathbf{e}_{t 2} .
\end{gathered}
$$

Предполагается, что $e_{z}$ направлен перпендикулярно поверхности подложки, или, если требуется связь, „В другую сторону“:

$$
\begin{gathered}
\mathbf{e}_{l}=\sqrt{\frac{3}{6}} \mathbf{e}_{x}+\sqrt{\frac{1}{6}} \mathbf{e}_{y}+\sqrt{\frac{2}{6}} \mathbf{e}_{z}, \\
\mathbf{e}_{t 1}=-\sqrt{\frac{3}{6}} \mathbf{e}_{x}+\sqrt{\frac{1}{6}} \mathbf{e}_{y}+\sqrt{\frac{2}{6}} \mathbf{e}_{z}, \\
\mathbf{e}_{t 2}=-\sqrt{\frac{4}{6}} \mathbf{e}_{y}+\sqrt{\frac{2}{6}} \mathbf{e}_{z} .
\end{gathered}
$$

Ортогональность тройки $e_{l}, e_{t 1}, e_{t 2}$ или тройки $e_{x}, e_{y}, e_{z}$ легко проверяется. Подставляя последние выражения в запись волнового вектора, имеем для его $x$ - и $y$-компонент:

$$
\begin{gathered}
k_{x}=\sqrt{\frac{3}{6}}\left(k_{0}+k_{l}\right)-\sqrt{\frac{3}{6}} k_{r} \cos \varphi, \\
k_{y}=\sqrt{\frac{1}{6}}\left(k_{0}+k_{l}\right)+\sqrt{\frac{1}{6}} k_{r} \cos \varphi-\sqrt{\frac{4}{6}} k_{r} \sin \varphi .
\end{gathered}
$$

В итоге для квадрата поперечного волнового вектора $k_{\perp}^{2}=k_{x}^{2}+k_{y}^{2}$, имеем выражение (3).

Если взять дифференциал от выражений для $k_{x}, k_{y}$ при фиксированной энергии $E$, получим

$$
\begin{aligned}
d k_{x}= & \frac{1}{\sqrt{2}}\left(1+\frac{m_{t} k_{l}}{m_{l} k_{r}} \cos \varphi\right) d k_{l}+\frac{1}{\sqrt{2}} k_{r} \sin \varphi d \varphi, \\
d k_{y}= & \frac{1}{\sqrt{6}}\left(1-\frac{m_{t} k_{l}}{m_{l} k_{r}}(\cos \varphi-2 \sin \varphi)\right) d k_{l} \\
& -\frac{1}{\sqrt{6}} k_{r}(\sin \varphi+2 \cos \varphi) d \varphi,
\end{aligned}
$$

При этом было использовано то, что $d k_{r}=$ $=-m_{t} k_{l} / m_{l} k_{r} d k_{l}$, с учетом выражения $k_{r}$ через $E$ и $k_{l}$ из соотношения $\hbar^{2} k_{l}^{2} / 2 m_{l}+\hbar^{2} k_{r}^{2} / 2 m_{t}=\left(E-E_{\mathrm{cS}}\right)$. На основе $d k_{x}, d k_{y}$ рассчитывается якобиан [8] преобразования от переменных $k_{x}, k_{y}$ к переменным $k_{l}, \varphi$ (формула (9) основного текста).

\section{Список литературы}

[1] A. Schenk. Advanced physical models for Silicon device simulations (Springer, Wien, NY., 1998) Chap. 5.

[2] М.И. Векслер, С.Э. Тягинов, Ю.Ю. Илларионов, Yew Kwang Sing, Ang Diing Shenp, В.В. Федоров, Д.В. Исаков. ФТП, 47 (5), 675 (2013).
[3] J. Robertson, R.W. Wallace. Mater. Sci. Eng. R, 88, 1 (2015).

[4] Y.Y. Illarionov, M.I. Vexler, S.M. Suturin, V.V. Fedorov, N.S. Sokolov, K. Tsutsui, K. Takahashi. Microelectron Eng., 88 (7), 1291 (2011).

[5] Yu.Yu. Illarionov, M.I. Vexler, M. Karner, S.E. Tyaginov, J. Cervenka, T. Grasser. Current Appl. Phys., 15 (2), 78 (2015).

[6] Crystals with the fluorite structure, ed. by W. Hayes (Clarendon Press, Oxford, 1974).

[7] L.F. Register, E. Rosenbaum, K. Yang. Appl. Phys. Lett., 74 (3), 457 (1999).

[8] И.Н. Бронштейн, К.А. Семендяев. Справочник по математике для инженеров и учащихся втузов (М., Наука, 1980) c. 380 [понятие ,якобиан“].

Редактор А.Н. Смирнов

\section{Modeling tunnel electron transport in a semiconductor-crystalline insulator-Si(111) system}

\section{M.I. Vexler}

loffe Institute,

194021 St. Petersburg, Russia

Abstract Tunneling carrier transport through a thin insulator (exemplarily $\mathrm{CaF}_{2}$ ) between the (111)-Silicon substrate and the semiconductor gate, is studied theoretically. Conservation of a transverse electron wave vector, which is large in value, and limitation on the available state density in the gate are considered. Due to a state-density-related restriction, the tunneling current at low insulator bias should be smaller than in similar structures with a metal gate. The same effect is responsible for a change in the energetic distribution of tunneling electrons, both for the case of a transfer between the conduction bands and when a transport occurs between the conduction band of $\mathrm{Si}(111)$ and the gate valence band. 